\title{
Survey of designing user interface for mobile applications
}

\section{Sarah Hamed Al Ahmadi *}

Department of Computing and Information, Community college, Taibah University, Medina, Saudi Arabia

\author{
Index Terms \\ User Interface \\ Design \\ Human-Computer Interaction \\ Usability \\ User-Centered Design
}

Received: 17 October 2016 Accepted: 17 January 2017 Published: 21 April 2017

\begin{abstract}
This search aims to explore the issues in developing the User Interface in mobile devices applications which is known as Human-Computer Interaction (HCI). The challenges that face the designers in developing the mobile UI are how to create usable and efficient UI and what are the guidelines or principles they should take into account to make interactive and interaction design. In addition, the software and hardware limitations they are encountered with, and also how they involve the users and their experience in designing process according to User-Centered Design (UCD) principles are considered. I gave the concepts for user interface, $\mathrm{HCI}, \mathrm{UCD}$, and usability issue and its relationship to designing process. Also I mentioned the general guidelines which are known as "Shneiderman's Eight Golden Rules of Interface Design" that should be considered. Moreover I explored that each platform nowadays has its own guidelines for best development of interaction process and that is why the applications of specific stage have the same layout. And I conclude that there is need for new methodologies and strategies of designing user interface that differs from methods used for developing traditional user interfaces due to enormous evolution of mobile devices and people's preference of such technology.
\end{abstract}

(C) 2017 The Author(s). Published by TAF Publishing.

\section{INTRODUCTION}

Improvements in mobile innovation have enabled a wide variety of applications to be produced that can be consumed by individuals in their daily life activities. Designers in some cases neglect the way that users will need to connect with such devices in diverse circumstances. Small screen sizes, high power utilization rates and constrained input modalities are only a percentage of the issues that emerge when planning applications for such gadgets. User interface where the interaction is done between people and programs plays an important role in designing mobile applications. Without a good design for the user interface the whole application is useless since its requirements are provided by its interface. There are many concepts that should be considered and understood in designing mobile user interface. Through my research in this field there is no completed paper that talks about all aspects associated with this kind of designing. Because of that, this paper aims to cover the important related aspects in this field. Designing mobile user interface required in-depth understanding of mobile attributes, achieving a good usability and considering user experience by applying user-centered design approaches. At the point when the amount of different properties and settings of the device is big, user interface loses its logicality and becomes complex and hard to use. In this paper, I talked about the challenges of mobile devices whether they face software challenges or hardware challenges. After that, user interface and its importance are explained. In the next sections, I defined the related concepts which are Human Computer Interaction, usability issues, user-centered design approaches, and the principles and the guidelines of designing the user interface.

\section{A. Mobile Devices and their Challenges}

These days the phones don't perform more than just calling function. Mobile devices become replacement of

\footnotetext{
* Corresponding author: Sarah Hamed Al Ahmadi

†Email: sarah.alahmadi90@gmail.com
} 
many daily activities such as workplaces or traveler guides and so on. According to [1], a mobile device is defined as a pocket-sized computing device that has a small display screen, a small keypad with mini buttons or a touch screen with stylus of input. Designing user interfaces for such devices causes many difficulties; some are hardwarerelated while the others are software-related. Software challenges are represented in hierarchical menus, navigating and browsing, and images and icons [1].

Taking the menus design in the desktop applications and applying it to a mobile application without a perfect understanding of the methods of inputs and outputs can cause an unproductive interaction design [1], [2]. The most effective alternative is hierarchical menus. A user then can pick out a menu element that can then display another submenu and so on till the user arrives the wanted task. The perfect hierarchy display with mobile devices is one with only four to eight items on each level and it is better to order in a hierarchy with more levels than in a hierarchy with more items per level [1]. With mobile devices that normally have small screens, presenting information that is designed for large screens has to be divided into small units to be suitable for small screen of mobile devices [3].

This process facilitates the navigation and browsing of the data. For images and icons such as drawings, diagrams, maps, and logos that are usually used in personal computers, visualization should be redesigned and to fit the capabilities and vision of mobile devices. On the other side, limited input and output manners and power consumption represent the major hardware challenges [1]. According to [4] there are three central input methods for mobile devices that are: the keyboard, the touch screen, and the scroll wheel. Hitting a key in keyboard style leads to perform a task or navigate through the different menus on screen. And hitting the touch screen lets a user do the diverse tasks. And the scroll wheel can be scrolled and pushed by a user to do a function and also navigate through the menus and submenus [1]. There are various output facilities that are used on mobile devices. The famous output styles in mobile devices are: small-sized screen which is the major one and the audio outcomes [1]. Screen size should be designed carefully to be suitable for the different types of mobile devices [4].

The audios are considered useful output for feedback messages to the user, and used in combination with the graphics and text messages to produce best interaction between the human and the devices [4]. Sound effects are sent as hidden messages to the user to some extent and a system feedback for some occurs [5]. Because mobility is a key feature of mobile devices, we should pay attention to meet that so they become easy to be held by the user [6]. To achieve that, power consumption management should be designed well [1]. [7] indicate that the power wasted by an application relies on the level of execution demanded by the application or user. They suggested a power management model that gives energy savings for the processor and optimizes energy savings for other devices such as display unit, $\mathrm{RF}$ unit, keyboard, and memory.

\section{USER INTERFACE DESIGN}

\section{A. The Concept of User Interface Design}

The revolution of mobile devices nowadays has made the entire user interface configuration experience a change. Small screen sizes and specific I/O devices of such gadgets set new requests and difficulties to user interface design [8]. The user interface paradigm in mobile application is based around widgets, touch, physical movement, and keyboards instead of the known WIMP (Windows, Icons, Menus, Pointer) interface style of Apple's iOS and Microsoft Windows [9]. The different mobile platforms contain their own UI libraries and rules so most of the native ("that run entirely on the mobile device") or web ("which have a small device-based client with execution occurring on remote server") applications will share a typical "look and feel" [9].

It's important for the application designer to commit to platform guidelines to meet the users' expectations. With the challenge of making the most ideal utilization of small screen space, user interface design takes significance than any other time. The users are usually looking to rapidly finish a particular task, and can't exploit the full scope of usefulness given by a traditional application that is found in the regular computers. Because of that most traditional programs should be redesigned to highlight the most generally used functions and to make best utilization of the screen and the mobile user interface paradigm [9].

\section{B. Human-Computer Interaction (HCI)}

According to [10], [11] and [12], HCI is concerned with the study of the relationship between individuals and mobile innovation and in addition, the methods of reaction, design practices, the apparatuses, procedures used, and the information that is obtained through these devices. It includes understanding of the users and their different abilities and anticipations and how these can be reflected 
in the user interface design [12]. The designer ought to understand what the user needs of mobile devices and what actions they need to perform when using them, and also what attributes of the users that could significantly affect their execution with such devices for instance, age or a physical disability such as blindness, all of these understanding sides refer to context of use [12]. All these aspects should be considered in designing process to meet users' needs. Visual deficiency. There are many disciplines that are taken into account when studying the mobile HCI.

These aspects according to [12] are a lot but the major of them are psychology, computer science, sociology, and design. Psychology has key influence on the HCI because research ways and system assessment techniques currently used in mobile HCI are borrowed from psychology approach. Computer science is in charge of supplying software tools to build the interfaces to the users. Sociology is responsible for discussing socio-technical sides of HCI. It explores the effect of mobile technology in social circumstances. People working in the design area are interested in the design outline of the interface such as colors and positioning of text or graphics on a screen. This is a critical area of mobile $\mathrm{HCI}$ because of the limited screen size available for most mobile devices.

\section{User Interface Usability}

Usability is a fundamental factor in the configuration user interface. Usability means whether an application is easy to learn, easy to use, and enjoyable to use for the users [12]. ISO organization introduces usability as system should be effective, efficient and satisfying for the interactive user [13]. Effectiveness identifies with how great the system is at what it should do, efficiency identifies whether or not the system performs users' tasks, and satisfaction measures individuals' impression of the system and the amount of ease it has [12]. Planning a graphical user interface with great usability is a challenge particularly when it is proposed to be kept running in devices with changing screen sizes and I/O gadgets [8].

For perfect usability according to [8], there are some instructions to be applied when designing user interface. The window size of the user interface ought to be kept small so the window can fit into a small display and be likewise utilized with low screen resolution. On the other hand interface components such as Toolbar buttons should be big and far enough to fit users' fingers, and also the number of them in a particular view is small. Users of the user in- terface are varying, there are old, young, users with special physical abilities, and so on.

All of them ought to be considered in designing process. One of these considerations is to design multiple user interface modes one as basic mode and the others as advanced modes or specified purposes. The basic mode should be public for all environments and includes most frequent and demanding actions for users. Beside multiple modes, for better designing of user interface each mode should be hierarchical structure which means it has multi views so the most common actions are existing in the main views and the infrequent commands available on sub-views. To increase simplicity and usability, users' input should be as hidden as possible by automating them on the different views.

\section{Usability Factors}

There are many usability models existing; one of them is PACMAD (People At the Centre of Mobile Application Development) which was proposed by [14]. Also there is ISO and Nielson [14] as examples. All of them state three factors of usability that affect the entire design of the user interface. These are user, task, and context of use. End users is significant factor and should be involved in designing process. User's previous experience affects the way of using the interface.

There are expert users and novel users, all prefer an interface that is intuitive and easy to cross over and which lets them to discover what they want. Task is the goal of the user they want to perform from specific application. Additional tasks can be added well without making the reach to original tasks difficult by applying a good usability model. Context of use on the other hand means the whole environment of using the application whether physical location or external interaction with this environment.

\section{E. Usability Attributes}

Beside the Effectiveness, Efficiency, and Satisfaction attributes mentioned above the PACMAD model, [14] added more attributes that are Learnability, Memorability, Errors and Cognitive load. Each of these characteristics has an influence on the complete usability of the system. Survey made by [15] detected that users will devote on average 5 minutes or less learning to use a mobile application. So Learnability measures how long a person takes to be able to use the application successfully. Memorability is the abil- 
ity of a user for how to use the software without the need to relearn it after a period of time. Errors permit developers to discover the most troubling ranges for users while using the application and how to enhance them and avoid them in next versions. Finally Cognitive load indicates to the amount of cognitive processing essential by the user to use the application.

\section{F. User-Centered Design (UCD)}

According to [16], UCD is defined as "a multidisciplinary design approach based on active involvement of users for a clear understanding of the user and task requirements, and the iteration of design and evaluation". To achieve a good usability, designers should choose a UCD approach to system development. There are four phases of UCD [12], [17]. First stage is understanding and identifying the context of use. The outputs of this phase provide developers with information that is used in design and evaluation processes. Secondly determine user and organizational requirements which specify characteristics and needs of the users. The output is a clear description of the application's tasks and interaction guide between the users and the system. Thirdly produce designs and prototypes to ensure that the system meets its requirements and gets users' feedback. Final stage is to evaluate the system based on users' assessment and include their feedback as early as possible.

\section{G. Guidelines of Designing User Interface}

The most famous guidelines of designing user interface is Shneiderman's “Golden Rules of Interface Design” [18]. It is applied rules in regular user interface and to be ideal for mobile user interface, it should have some modifications. These modified guidelines according to [19] are:

- Enable frequent users to use shortcuts: Since time is frequently more crucial to a mobile device, decreasing the quantity of operations expected to perform tasks is a key factor in the ease of use of mobile devices.

- Offer informative feedback: For each activity, there ought to be some system reaction, for example, a beep while squeezing a key or a fault message for an invalid data value.

- Design dialogues to yield closure: Groupings of activities should be sorted into collections with a starting, center, and end. Users ought to be given the satisfaction of achievement and completion.

- Support internal locus of control: Users need to be accountable for the system and have system's reactions to their activities rather than feeling that the system is controlling them. User interface ought to be designed such that users start activities rather react to them.

- Consistency: Mean developer should take into account the consistency over different stages and gadgets for the same application. Users might need to switch between their desktop computers and diverse mobile devices. In this circumstance, consistency ought to be kept up in the middle of desktop and mobile devices. Consistency can likewise be accomplished by making I/O approaches that are gadgetfree.

- Reversal of actions: Permitting simple inversion of activities may be more challenging for mobile devices due to an absence of resources and processing power. Such devices have less memory to store the conditions of past actions.

- Error prevention and simple error handling: Avoiding and control of errors on mobile interfaces are like those for desktop interfaces, despite the fact that the need turns out to be more basic because of the more fast actions in such environment. Errors handling likewise need to take the physical configuration of mobile devices into account.

- Reduce short-term memory load: Given the constraints of a user's short-term memory, interfaces ought to be designed such that small memorization is required among the execution of tasks.

\section{DISCUSSION}

User interface design is an important field to be searched due to its role of providing the different functions of an application. Poor designing of user interface leads to useless application even if it has a creative idea. Configuring user interface has many issues to be considered and to get beneficial interface. Through my survey there are a lot of researches on developing a good user interface in regular applications but for mobile applications, there is a view and there is no completed research covering all designing aspects. In this paper, I encompass all related sides in designing process. First, designers ought to be aware of the physical structure and constraints of mobile devices.

The major physical limitation that all researchers agreed with its high influence is the limited screen size of such devices beside the restricted input and output methods. It affects the usability factor, HCI methodologies, and UCD approaches. The limited screen size makes it hard to efficiently view information and navigate easily through the information. Furthermore, dealing with the limited battery power and managing power consumption has also become one of the major important issues for system design- 
ers. Likewise there are software difficulties that emerge due to the physical restrictions. Menus, browsing methods, and icons styles used in regular interfaces are difficult to be applied in mobile interface. So there is need to redesign all these factors to fit to mobile devices. Hierarchical menus is best alternative of menus and more hierarchical levels better than less levels with more items each. Moreover the fragmented information into small parts gives typical browsing method and facilitates the navigation through the small-sized screen. HCI which is the science that has interest in interaction between devices and people, has significant role and should be considered in designing interfaces. HCI defines all related sides that affect the designing.

This includes understanding the users, their needs, and their behavior and psychology and in different way it determines the context of use. As a real example of understanding the user behavior, I will make simple comparison between Return Button on Apple devices and Samsung devices. In Apple IOS, we found it on the upper left corner of the screen while in Android OS, it is on bottom right corner. Users usually prefer to use the thumb to do most tasks, so we prefer always the tasks to be on the bottom of the screen. As a result of this comparison, I found that the Samsung devices excel the Apple devices in this specific aspect. Good HCI approach leads to best usability of the interface which is simply measure of application, does it meet its requirements? Does it perform tasks of the users right? And does it provide the functions in easy and attractive manner? From the previous discussion, I noted that developers focus to include the users in designing user interface especially in mobile devices due to its increasing growth. As a result UCD is a methodology that makes the users part of designing procedure to get their impressions and feedback during developing interface that is done in iterations for best results. Finally as we note nowadays, there are three basic operating systems for mobile devices having IOS, Android, and windows phone. Each of them has its own guidelines of developing user interfaces [21], [22], [23], [24], [25]. Applications for each of these platforms have common features.

As an example if we look at IOS applications we can easily note the unified layout for them. They all have Status Bar at the top of the screen which usually contains the important information about the device. Also for navigation process through some application information, there is navigation bar which is at the top of the screen and below status bar. And for application's tasks, there is toolbar which appears at the bottom of the application and contains all the actions provided by that application. And if we look at fea- tures of other platform applications, we will see the similarity of their layout.

\section{CONCLUSION}

I discussed in this paper the most aspects related to designing user interface for mobile applications while there is no paper encompassing them before. I gave the concepts for user interface, HCI, UCD, and usability issue and its relationship to designing process. Also I mentioned the general guidelines which are known as "Shneiderman's Eight Golden Rules of Interface Design" that should be considered. Moreover I explored that each platform nowadays has its own guidelines for best development of interaction process and that is why the applications of specific stage have the same layout. And I conclude that there is need for new methodologies and strategies of designing user interface that differs from methods used for developing traditional user interfaces due to enormous evolution of mobile devices and people's preference of such technology.

\section{REFERENCES}

[1] K. Y. Huang, "Challenges in human-computer interaction design for mobile devices," in Proceedings of the World Congress on Engineering and Computer Science, San Francisco, SA, 2009.']

[2] H. Sharp, Y. Rogers and J. Preece, Interaction Design Beyond Human-Computer Interaction. New York, NY: John Wiley \& Sons, 2015.

[3] L. Wang and A. S. M. Sajeev, "Roller interface for mobile device applications," in Proceedings of the Eight Australasian Conference on User Interface, Victoria, Australia 2007.'

[4] M. A. Muhanna, "Exploration of human-computer interaction challenges in designing software for mobile devices," Thesis, University of Nevada, Reno, NV, 2007.

[5] H. Korhonen, J. Holm and M. Heikkinen, "Utilizing sound effects in mobile user interface design," in Human-Computer Interaction-Interact, Lecture Notes in Computer Science, vol. 4662, C. Baranauskas P. Palanque J. Abascal and S. D. J. Barbosa, Eds. Berlin, Germany: Springer, 2007

DOI: $10.1007 / 978-3-540-74796-3 \_27$

[6] B. A. Myers, J. Nichols, J. O. Wobbrock and R. C. Miller, "Taking handheld devices to the next level," Computer, vol. 37, no. 12, pp. 36-43, 2004.

DOI: $10.1109 /$ MC.2004.258 
1[7] H. S. Ashwini, A. Thawani and Y. N. Srikant, "Middleware for efficient power management in mobile devices," in Proceedings of the 3rd International Conference on Mobile Technology, Applications \& systems ( $\mathrm{p}$. 49), Bangalore, India, 2006.

१[8] R. Luostarinen, J. Manner, J. Määttä and R. Järvinen, "User-centered design of graphical user interfaces," in Military Communications Conference, San Jose, CA, 2010.

I [9] A. I. Wasserman, "Software engineering issues for mobile application development," in Proceedings of the FSE/SDP Workshop on Future of Software Engineering Research, Moffett Field, CA, 2010. DOI: $10.1145 / 1882362.1882443$

I [10] A. D. E. L. E. Botha, D. A. R. E. L. L. E. Van Greunen and M. A. R. L. I. E. N. Herselman, "Mobile humancomputer interaction perspective on mobile learning," in 6th Annual International Conference on Computing and ICT Research, Kampala, Uganda, 2010.

१[11] B. Bauer and A. S. Patrick, "A human factors extension to the seven-layer OSI reference model," [Online]. Available: https://goo.gl/83Dy9p

$1[12]$ S. Love, Understanding Mobile Human-Computer Interaction. Amsterdam, Netherlands: Elsevier, 2005.

1[13] A. Abran, A. Khelifi, W. Suryn and A. Seffah, "Usability meanings and interpretations in ISO standards," Software Quality Journal, vol. 11, no. 4, pp. 325-338, 2003.

१ [14] R. Harrison, D. Flood and D. Duce, "Usability of mobile applications: Literature review and rationale for a new usability model," Journal of Interaction Science, vol. 1, no. 1, pp. 1-16, 2013.

१[15] D. Flood, R. Harrison, C. Iacob and D. Duce, "Evaluating mobile applications: A spreadsheet case study," International Journal of Mobile Human Computer Interaction (IJMHCI), vol. 4, no. 4, pp. 37-65, 2012

[16] A. Kushniruk, H. Monkman, E. Borycki and J Kannry, "User-centered design and evaluation of clinical information sys tems: A usability engi neering perspective," in Cognitive Informatics for Biomedicine, V. L. Patel, T. G. Kannampallil and D. R. Kaufman Eds. Berlin, Germany: Springer International Publishing, 2015.

1[17] International Organization for Standardization, "Human-Centred Design Processes for Interactive Systems," Ergonomics of Human-System Interaction, ISO, Geneva, Switzerland, 1999.

I[18] B. Shneiderman, Designing the User Interface: Strategies for Effective Human-Computer Interaction, Vol. 3. Reading, MA: Addison-Wesley, 1992.

1[19] J. Gong and P. Tarasewich, "Guidelines for handheld mobile device interface design," in Proceedings of DSI Annual Meeting (pp. 3751-3756). Boston, MA, 2004.

१[20] J. Larysz, M. Němec and R. Fasuga, "User interfaces and usability issues form mobile applications," in Digital Information Processing and Communications. Berlin, Germany: Springer Berlin Heidelberg, 2011.

1 [21] Developer, "Interface user; Android developers,"

[On- line]. Available: Developer.android.com

[22] Developer, "Ios human interface guidelines: Designing for Ios," [Online]. Available: https://developer.apple.com/

[23] WindowsPhone7, "Windowsphone 7,- chapter 2: User interface guidelines," [Online]. Available: https://goo.gl/Vuqv6V

[24] F. J. Yang, "The user interface design of an intelligent tutoring system for relational database schema normalization," International Journal of Technology and Engineering Studies, vol. 2, no. 3, pp. 70-75, 2016. DOI: $10.20469 /$ ijtes.2.40002-3

[25] O. A. Osahenvemwen "Evaluation of mobile communication network performance," International Journal of Technology and Engineering Studies, vol. 3, no. 1, pp. 09-19, 2017. DOI: 10.20469/ijtes.3.40002-1 